\title{
The Practical Management of the
}

\section{Recalcitrant Tuberculous Patient}

\author{
BY CEDRIC NORTHROP, M.D., JOHN H. FOUNTAIN, M.D., \\ and DANIEL W. ZAHN, M.D.
}

Prevention of the development of recalcitrance is the logical beginning of practical management of tuberculous patients who fail to follow medical recommendations. Several steps can be taken to assist in lessening the uncooperative behavior of these patients.

\section{Sanatorium Care}

The first step in the prevention of recalcitrance is to have enough sanatorium beds available for the immediate care of all persons who have active tuberculosis. These beds should be located in reasonably attractive, conveniently located sanatoriums, staffed by competent and sympathetic personnel. To direct any great effort toward coercion of the uncooperative patient when there are cooperative patients awaiting hospitalization seems to be a questionable procedure.

Waiting lists for sanatorium care were abolished in Washington in 1948. As a result of

Dr. Northrop is tuberculosis control officer, Washington State Department of Health; Dr. Fountain is director of the division of tuberculosis control, Seattle City-King County Department of Public Health; and Dr. Zahn is chief of the medical service at Firland Sanatorinam, Seattle, Wash. This paper was presented before the forty-eighth annual meeting of the National Tuberculosis Association, Boston, May 27, 1952. building programs in Spokane, Yakima, and Tacoma, plus the acquisition of a surplus military hospital in Seattle, 2,400 beds, exclusive of those in Federal and mental institutions, are now available for the care of Washington tuberculosis patients. This number, divided by the 310 tuberculosis deaths of Washington residents (provisional data for 1951), establishes a ratio of 7.7 available beds' per annual death. The average number of patients hospitalized in 1951 was $2,100-a$ ratio of 6.7 patients per annual death.

\section{Patient Orientation}

Adequate indoctrination of the patient prior to admission to a sanatorium is also fundamental. The physician who makes the diagnosis of tuberculosis and refers the patient for sanatorium care will need to take the time to explain skillfully and in simple language the significance of the patient's individual tuberculosis problem and the steps he must take both to get well and to protect his family and his community. The assistance of public health nurses and medical social workers is valuable to reinforce this preliminary indoctrination.

However, a physician cannot delegate his full responsibilities to his assistants. He himself must start the education of the patient. Following preliminary discussion of the medical facts by the patient and his physician, the clinician refers the patient to the medical social service department and the public health nurses 
for further help. The support of the family, the care of the children or of the farm, and many other problems are reviewed and careful plans are made to meet the various situations that will arise during sanatorium treatment.

In many areas in the United States, the financial eligibility provisions written into the tuberculosis control law are stumbling blocks to sanatorium placement. The Washington statute states simply that the arrangements for hospital care for tuberculous patients shall be the responsibility of the jurisdictional health offcer, and this officer shall also have responsibility for determining the financial eligibility of patients admitted to tuberculosis hospitals. Since no standards for admission are written into the law, the health officer may concentrate on the epidemiological implications of the case rather than consider primarily the fiscal aspects. In other words, tuberculosis hospitalization becomes a part of a communicable disease control program rather than a component of medical care for the indigent. To the best of our knowledge, no tuberculosis patient needing sanatorium care in our State is staying at home because of financial obstacles.

\section{Prevention in the Sanatorium}

The next step in preventing the development of uncooperative patients takes place in the sanatorium. An interested, sympathetic medical staff, sufficient in number, is necessary. At Firland Sanatorium, Seattle, there is a staff of 19 full-time physicians to serve the $1,058 \mathrm{pa}$ tients now in residence- 55 resident patients per physician. This number of physicians is exclusive of interns, residents, pathologists, surgeons, or of the many part-time consultants. The medical staff is supplemented by five auxiliary services: education, occupational therapy, recreation, vocational guidance, and medical social service.

\section{Irregular Discharges}

In spite of adequate facilities for their care, many tuberculous patients refuse to enter the sanatorium, and a considerable number of those who do enter become irregular discharges (against medical advice, absent without leave, disciplinary). At Firland these irregular discharges constituted 47 percent of all discharges (exclusive of deaths) in 1948; 44 percent in $1950 ; 37$ percent in 1951 ; and 36 percent the first 4 months of 1952.

In 1949 Drolet and Porter (1) reported a detailed study of patients discharged in 1947 from tuberculosis institutions serving the New York metropolitan area. Of 1,413 discharges (deaths excluded), 731 (52 percent) were irregular. Apparently, this problem is severe in other seaport cities besides Seattle.

Analysis of the irregularly discharged patients at Firland Sanatorium in 1950 shows that 29 percent were alcoholics, 8 percent had obvious psychiatric difficulties, and 9 percent had records of jail sentences. In spite of the availability of adequate sanatorium care, slightly more than one-third of the discharges (other than patients who died) have been without medical approval. The magnitude of the problem has been recognized by health authorities, and some steps have been taken to reduce it.

\section{Legal Authority}

The first step was to strengthen the authority of the health officer in handling the uncooperative patient with communicable tuberculosis. The Washington law (Remington's Revised Statutes, sec. 6094, enacted in 1903) states in part: ". . Every health officer shall have the power to remove to and restrain in a pesthouse or isolation hospital, or to quarantine or isolate, any person sick with any dangerous, contagious, or infectious disease until such sick person shall have thoroughly recovered."

To define the power of the health officer further, on December 4, 1948, the Washington State Board of Health adopted the following regulation on pulmonary tuberculosis:

Isolation. Of such active cases as do not observe the precautions to prevent the spread of the disease. The place of isolation to be in such quarters as designated appropriate by the jurisdictional health officer, and for such time as necessary until one of the following conditions is fulfilled: (1) The patient's pulmonary disease is considered to be "apparently arrested" (National Tuberculosis Association Classification-1940); (2) the patient agrees to accept routine sanatorium care; (3) the patient dies; (4) other arrangements for adequate isolation are made, which, 
in the opinion of the jurisdictional health officer, protect the public from the spread of his infection.

Quarantine. Whenever, in the opinion of the jurisdictional health officer, he deems it necessary in the interest of the protection of the public, in uncooperative cases who refuse to observe precautions recognized as necessary to prevent the spread of this disease, quarantine procedures should be employed in accordance with the provisions under "isolation."

The deputy prosecuting attorney of King County was exceedingly helpful in specifying to the health officer the exact steps to be followed to conform to the principles of sound legal procedure. The first isolation order was issued by the King County Health Department on October 7, 1948; the first court order of commitment in King County for violation of an isolation order was issued February 4, 1949.' .

\section{Locked Ward}

As the compulsory isolation procedure was applied, it became apparent that a locked ward was necessary to handle the difficult individuals at Firland. Such a ward, of 27 beds, was made available at the sanatorium June 21, 1949.

The usual practice has been to keep the patient in the locked ward for 2 weeks after admission, and then transfer him to the regular wards of the hospital, the length of his stay there to depend on good behavior. If he disregards the institutional rules, gets drunk, or leaves the sanatorium, he is subject to reassignment to the locked ward for a longer time. Because of the admission of a few intractable patients, several of whom tried to set the ward on fire, experienced officials of the Seattle city police and fire departments. were consulted. These officials helped to promulgate security regulations for safer operation of the locked ward.

Once the locked ward had been established and the procedure for its use simplified, the number of patients under isolation order increased markedly. As of May 15, 1952, there were 118 persons at Firland under isolation order, 12 in the locked ward and 106 in regular wards with the same privileges as other patients, except that if they violate hospital rules or run away, back to the locked ward they go. Men patients requiring isolation predominate in the ratio of 5 to 1 . Four other sanatoriums in
Washington have set up locked wards varying in size from two to nine beds.

As the result of having adequate sanatorium facilities available, many of the small and medium-sized counties do not have one case of active tuberculosis known to the local health department in which the patient is staying at home against the recommendation of the health officer.

\section{Emotional Problems}

It was apparent by 1948 that many of the patients who were irregularly discharged had emotional problems of varying intensity, some of them frank psychoses. The development of the auxiliary services-education, occupational therapy, recreation, vocational guidance, and medical social service-is a constructive step forward. These services are helpful in creating a successful adjustment to sanatorium life for many patients.

However, they are not enough. There remains a group of patients unable or unwilling to comprehend the benefits of the sanatorium căre provided for them. We have undertaken several services in an attempt to meet these needs. One of the first steps was the formation of a chapter of Alcoholics Anonymous, with regular meetings in the sanatorium. The first meeting was held September 20, 1950, and meetings have continued, with an average attendance of approximately 20 members.

We hope that the provision of psychiatric consultation may help to combat irregular discharges. In cooperation with the University of Washington Medical School, psychiatric consultation services were expanded in 1949. Based on 2 years of experience, the consulting psychiatrist has formed some ideas on the management of maladjusted sanatorium patients, among whom alcoholics predominate.

Participation in Alcoholics Anonymous is very useful for the patients with mild to moderate maladjustment and can successfully tide many of them through their period of rest regimen. For the severe alcoholic, psychotherapy is difficult and often hopeless. Compulsory isolation and segregation are necessary if the patient and his community are to be protected. Some psychiatrists have maintained 
that this forcible isolation of recalcitrant tuberculous patients would create a group of bitter and highly antagonistic people. Our experience indicates that most of the patients isolated under compulsory quarantine have proved to be tractable and capable of being managed successfully, once they learn that they can be restrained successfully if they fail to cooperate.

One of the most valuable byproducts of the psychiatric consultation service is the education of the sanatorium medical staff on day-to-day evaluation and management of adjustment problems of patients. The value of this service cannot be overemphasized.

\section{$X$-raying Jail Population}

Edwards and Reisner (2) reported in 1940 on the value of $\mathrm{X}$-raying the jail population in New York City. In Washington several partial surveys were done in jails and one fairly complete, continuous survey in the Spokane County jail, which is located close to the health department, where miniature film equipment was available.

An X-ray machine was installed in the county jail in Seattle in June 1951 for routine Xraying of prisoners on admission. The group having the largest number of active cases was made up of the prisoners being sent to the county farm for rehabilitation of alcoholics. Of the 510 prisoners in this group, 88 (17.2 percent) were found to have active tuberculosis.

Case-finding efforts in jails are very lucrative in terms of cases found per amount of money spent. However, they do make absolutely necessary the provision of a locked ward for the prisoners discovered to have active tuberculosis. A prisoner may be sent to the sanatorium while awaiting trial or, if he has been tried and found guilty, the judge may suspend sentence provided the prisoner goes to the sanatorium for treatment. If the security facilities of the sanatorium locked ward are not adequate, there are single rooms in the county jail and a tuberculosis ward in the State penitentiary at Walla Walla.

\section{Management Possible}

Much experience has been gained during the past 3 years concerning the recalcitrant tuber- culosis patient and his management. Among the large number of recalcitrant individuals handled during this period, the Seattle CityKing County Health Department has had to secure only 16 warrants for the arrest of individual patients. Only 12, plus 2 repeaters, have been tried in court; all others have been handled through health department isolation regulations. The last instance in which it was necessary to go to court was on January 17, 1951manifestation of the fact that the local health department and the sanatorium are now "on top of the problem" and the group of individuals who are headstrong or distorted in their thinking can be handled successfully.

If the ultimate objective of wiping out tuberculosis is ever attained, it will be necessary for every metropolitan area to seek cases "on the other side of the tracks," and for every city to have a case-finding program in jails, prisons, and the flophouse district. In consequence it will be mandatory to set up in every city a facility for the detention of this type of patient. The epidemiological benefits will justify all the trouble; the expense is modest.

It is true that in the first 4 months of 1952 the irregular discharges still constituted 36 percent of all discharges (exclusive of deaths) from Firland. However, most of these patients were individuals who had progressed with their treatment to the point where they were ambulatory or semiambulatory, and merely left the sanatorium before fulfilling the recommended standard of 4 hours of work tolerance for 3 months. The health department usually rounds up fairly promptly patients who are sputumpositive and constitute a definite public health menace. These patients immediately return to the sanatorium or are sent back under a health department isolation order.

In King County (population 760,000), on May 20, 1952, three patients with active, communicable tuberculosis were known by the health department to be at home contrary to the wishes of the department. An additional 60 patients with communicable tuberculosis were at home, but their cooperation with the health department and their circumstances were such that they were not considered a public health menace. None was working and none had children at home. 


\section{Summary}

1. The practical management of the recalcitrant tuberculous patient begins with prevention of uncooperative behavior. Especially important is sanatorium care that is adequate both quantitatively and qualitatively.

2. The more nearly complete the job of tuberculosis control becomes, the higher the percentage of patients who are difficult to manage.

3. To solve the problem of the recalcitrant tuberculous patient two things are needed: $(a)$ adequate laws requiring isolation of the patient; (b) a place for forcible isolation, preferably where treatment can be given.
4. Of the 1,058 patients now at Firland Sanatorium in Seattle, Wash., 118 (11 percent) are under isolation order. Forcible isolation of these patients has not developed a group of bitter, antagonistic individuals; most of them become tractable and readily manageable.

\section{REFERENCES}

(1) Drolet, Godias J., and Porter, Donald E.: Why do patients in tuberculosis hospitals leave against medical advice? New York, N. Y., New York Tuberculosis and Health Association, 1949.

(2) Edwards, H. R., and Reisner, D.: Tuberculosis case finding. Studies in mass surveys. Am. Rev. Tuberc. 41: 1-159 (Supp. June 1940).

\section{Rabies Conference}

The South Middle Atlantic Regional Rabies Conference, attended by delegates from Pennsylvania, Ohio, Maryland, Delaware, Virginia, West Virginia, Tennessee, Kentucky, North Carolina, South Carolina, Georgia, and the District of Columbia, was held June 2-3 in Washington, D. C. Among the recommendations made were these: Each State should establish a rabies control program to be carried out through a committee composed of representatives from State agencies; provisions should be made for exchange of rabies control information between States; and each State should arrange for the prompt reporting of rabies cases in all animals.

The conference, called by the Public Health Service staff of the Region III office of the Federal Security Agency and co-sponsored by the Fish and Wildlife Service of the Interior Department and the Bureau of Animal Industry of the Department of Agriculture, stressed the need for dog immunization clinics and for the control of stray dogs. It called attention to the increase of rabies in the wildlife in this area, particularly in foxes and skunks, and the increased dangers of infection in cattle and such domestic animals as cats and dogs.

An Eastern Regional Rabies Council, a continuing body representing the States which took part in the conference, was established to develop plans and procedures to put the recommendations into effect. 